\title{
COMMENTS
}

\section{Comment on "Determination of first and second magnetic anisotropy constants of magnetic recording media"'[Appl. Phys. Lett. 77, 1689 (2000)]}

\author{
A. Lisfia) and J. C. Lodder \\ Information Storage Technology Group, $\mathrm{MESA}^{+}$Research Institute, University of Twente, P. O. Box 217, \\ 7500 AE Enschede, The Netherlands
}

(Received 21 March 2001; accepted for publication 19 July 2001)

[DOI: $10.1063 / 1.1402635]$

Recently Endo et al. ${ }^{1}$ proposed a new method in order to estimate the first and the second orders $\left(K_{1}\right.$ and $\left.K_{2}\right)$ of magnetocrystalline anisotropy in CoCrPtTa thin film media with full in-plane easy axis. The principle of their method is simple and consists of measuring the magnetization along the hard axis (see Fig. 1). By considering the first and the second orders of magnetocrystalline anisotropy, which is well justified in this alloy (CoCrPtTa) due to its hexagonal close-packed (HCP) structure, the total energy density in the configuration shown in Fig. 1 can be given by: $E_{T}=\left(E_{S}\right.$ $\left.+K_{1}\right) \sin ^{2}(\theta)+K_{2} \sin ^{4}(\theta)-H M_{s} \sin (\theta)$, where $E_{s}$ is the shape anisotropy of the thin film and it is equal to $\pm 2 \pi M_{s}^{2}$ for in-plane or perpendicular media. The angular position of $M_{s}$ can be determined by minimizing the total energy density: $\partial E_{T} / \partial \theta=\cos (\theta)\left[2\left(E_{s}+K_{1}\right) \sin (\theta)+4 K_{2} \sin ^{3}(\theta)-H M_{s}\right]=0$. The magnetization $M$, which is accessible by the measurement, is the projection of $M_{s}$ following the field direction $\left[M / M_{s}=\sin (\theta)\right.$, see Fig. 1]. By including this last formula in that of the equilibrium position of $M_{s}$, the following equation can be easily established: $H / M=a M^{2}+b$, where $a$ and

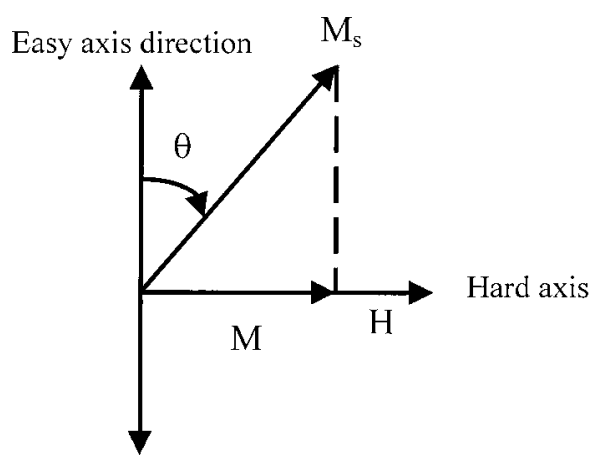

FIG. 1. Configuration for measuring $K_{1}$ and $K_{2}$ for a system with uniaxial anisotropy. The magnetization is measured along the hard axis. $b$ are two constants depending on the second and first order, respectively, $\left(a=4 K_{2} / M_{s}^{4}, b=\left(2 E_{s}+K_{1}\right) / M_{s}^{2}\right)$. By measuring the ratio $H / M$ and plotting it versus the square of the magnetization $\left(M^{2}\right)$, Endo et al. ${ }^{1}$ estimated the first and the second orders of anisotropy at different temperatures.

The most important requirement for this method is the perfect orientation of the anisotropy, which is well illustrated in the CoCrPtTa films studied. Both x-ray diffraction and magnetization curve of Fig. 3 in Ref. 1 confirm the good orientation of the easy axis parallel to the film plane. All these conditions suggest that these magnetic films (CoCrPtTa) constitute an ideal system for the application of this method.

However, in our opinion this method is not new and is already known for a long time. ${ }^{2}$ To our knowledge, Sucksmith and Thompson were the first authors to propose this method and use it to estimate the anisotropy constants (first and second order) for HCP as well as fcc cobalt at different temperatures. Additionally, in contrast to the author revelations, this method is not accurate, especially in the determination of the second order $K_{2}$ for polycrystalline material. The differentiation of the slope (a) from which $K_{2}$ is deduced allows us to establish the following relation: $\Delta K_{2} / K_{2}$ $=\Delta a / a+4 \Delta M_{s} / M_{s}$. If we consider in the extreme case that there is no error in the measurement of the slope a ( $\Delta a=0)$, the other source of the error can be the saturation magnetization. Usually it is difficult to estimate precisely $M_{s}$ in the thin films due to inaccuracy in the thickness measurement. If we assume in the best case that the relative error in the thickness is about $5 \%, \Delta K_{2} / K_{2}$ should be larger than $20 \%$. With this method, the first order $K_{1}$ can be estimated more accurately than $K_{2}$ because of its dependence on $M_{s}^{2}$.

${ }^{1}$ Y. Endo, O. Kitakami, S. Okamoto, and Y. Shimada, Appl. Phys. Lett. 77, 1689 (2000).

${ }^{2}$ W. Sucksmith and J. E. Thompson, Proc. R. Soc. London, Ser. A 255, 362 (1954)

${ }^{a)}$ Electronic mail: a.lisfi@el.utwente.nl 